\title{
Radiological analysis of the position of epidural catheters in the thoracic epidural space
}

\author{
Mun Gyu Kim, Si Young Ok, Se Kwang Park, Ho Bum Cho, and Sang Ho Kim \\ Department of Anesthesiology and Pain Medicine, Soonchunhyang University Seoul Hospital, Seoul, Korea
}

Thoracic epidural catheterization is used widely for anesthesia and postoperative pain control in thoracic and abdominal surgery. Generally, an epidural catheter is inserted only $4-5 \mathrm{~cm}$ into the epidural space, as excessive catheter insertion causes complications like vascular injury and paresthesias [1]. Nevertheless, a catheter can often be inserted quite a distance as a consequence of the catheter becoming dislodged outside the epidural space with changes in the patient's position. Most studies of the amount of epidural catheter insertion have focused on the lumbar level $[2,3]$. Therefore, we examined catheter advancement at the thoracic level.

This study enrolled 72 mastectomy patients graded as American Society of Anesthesiologists class I or II and treated under general or epidural anesthesia. A thoracic epidural catheter was inserted for post-surgical pain control. Patients with coagulopathy, infection at the needle puncture site, a history of spine surgery, or scoliosis were excluded. The aims of the study were explained to the patients and the study was approved by the Institutional Review Board.

In a sitting position, the patient's neck was flexed as much as possible while the patient held the opposite knee with both hands, and the inferior tips of both scapulas were palpated to find the seventh thoracic spinous process. Then, an epidural puncture was made in the largest interspinous space out of T3-4, T4-5, or T5-6. Using a midline approach, with the bevel of a 17-gauge Tuohy needle facing cephalad, the needle was inserted and the epidural space was confirmed by the loss-ofresistance method using air. Then, a 19-gauge end-hole epidural catheter (Flextip Plus ${ }^{\mathrm{TM}}$ Epidural Catheter; Arrow International
Inc., Reading, PA, USA) with a spring wire coil was inserted into the epidural space. If the patient complained of severe pain, insertion was stopped and the catheter was fixed. If the patient did not complain, the catheter was inserted a maximum of $8 \mathrm{~cm}$ into the epidural space. Then, anteroposterior and oblique X-rays of the thoracic spine were taken. The catheter was fixed $4 \mathrm{~cm}$ into the epidural space, and then local anesthetics were administered into the epidural space and the surgery was performed. From the thoracic X-ray images, the shape of the epidural catheter was classified as A) straight, B) a single loop, C) a double loop, or D) coiled (more than two loops) (Fig. 1). The maximum inserted length of the catheter into the epidural space was recorded as the catheter tip position for a straight catheter or otherwise the higher of the catheter tip or the kinked point at the highest position. It was also noted whether the catheter tip was below the epidural puncture site. All assessments were made by three anesthesiologists and the length of catheter inserted was recorded in units of 0.5 segments.

The thoracic epidural catheter was straight in $8(11.1 \%)$ subjects, a single loop in 23 (31.9\%), a double loop in 31 (43.1\%), and coiled in $10(13.9 \%)$. The catheter was inserted into the epidural space a mean of $1.85 \pm 1.13$ (range $0-5$ ) segments. In no case was the catheter tip located below the site of epidural puncture.

Using a midline approach, when a catheter was inserted into the lumbar epidural space in 40 patients until it started to coil, Kim et al. [2] reported that the catheter was inserted $2.0-3.5 \mathrm{~cm}$ in $29,<2 \mathrm{~cm}$ in 4 , and $>3.5 \mathrm{~cm}$ in 7 , including one patient in whom it was inserted $8 \mathrm{~cm}$. In two patients (5\%), the catheter

Corresponding author: Si Young Ok, M.D., Ph.D., Department of Anesthesiology and Pain Medicine, Soonchunhyang University Seoul Hospital, 59, Daesagwan-gil, Yongsan-gu, Seoul 140-743, Korea. Tel: 82-2-709-9302, Fax: 82-2-790-0394, E-mail: syok2377@naver.com

(c) This is an open-access article distributed under the terms of the Creative Commons Attribution Non-Commercial License (http:// creativecommons.org/licenses/by-nc/3.0/), which permits unrestricted non-commercial use, distribution, and reproduction in any medium, provided the original work is properly cited. 

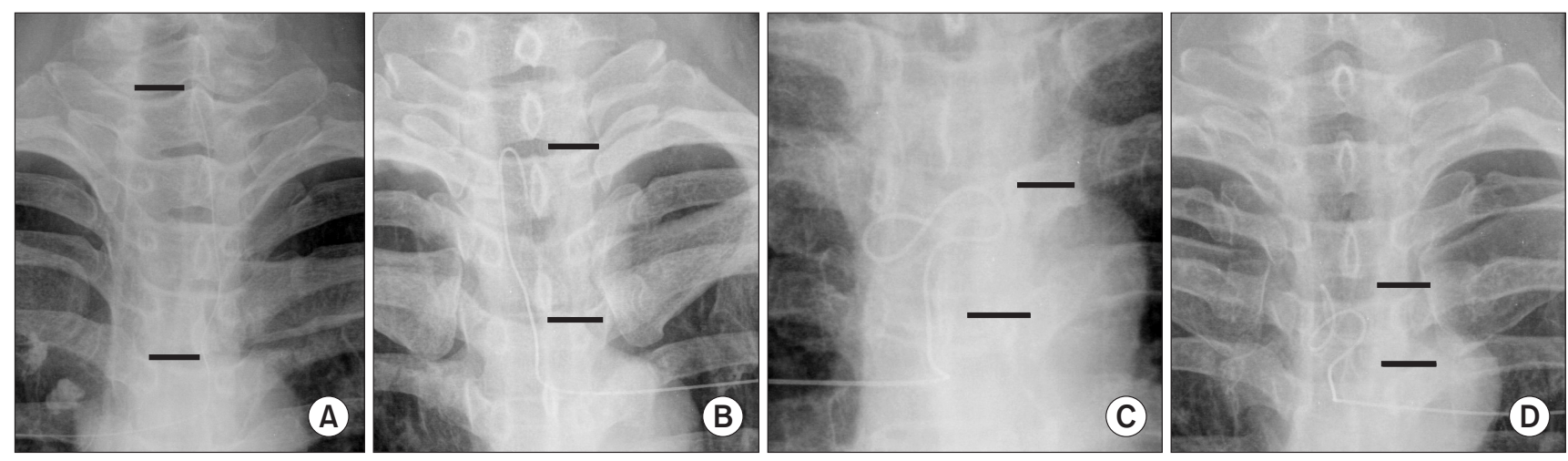

Fig. 1. The shapes, puncture sites, and insertion lengths of the catheters. The length is the distance between the black bars. (A) straight, (B) single loop, (C) double loop, and (D) coiled. (A) T4-5 (4.0 segments), (B) T3-4 (2.0 segments), (C) T5-6 (1.5 segments), (D) T4-5 (1.0 segment).

was inserted in the direction opposite the Tuohy needle bevel unintentionally. In their series, Bridenbaugh et al. [3] reported that $14 \%$ of patients had the catheter inserted without coiling, while $86 \%$ had coils (62\%) or a single loop $(24 \%)$ at the insertion site when the catheter was inserted about $5 \mathrm{~cm}$ into the lumbar epidural space.

In our series, 8 subjects (11.1\%) had a catheter inserted without coiling, while 41 (57\%) had a coiled or double looped catheter. In no case was the catheter inserted in the direction opposite the needle bevel. In 16 subjects (22\%), the catheter was inserted 3.0 or more segments.

An epidural catheter is prevented from moving cephalad by connective tissue, blood vessels, and nerve roots in the epidural space [1-3]. Especially in the lumbar region, moving cephalad is difficult because the Tuohy needle is almost perpendicular to the skin. In addition, the approach (midline or paramedian) used with a Tuohy needle [4] or the material that the epidural catheter is made from [5] can affect catheter insertion.

This study adds to the little information available on catheter insertion at the upper thoracic level. One possible error in the analysis is that we did not identify the relationship between the catheter tip position and puncture site in real time. Rather, we recorded the deepest location of the catheter tip or catheter loop formation after catheter insertion. In addition, although catheter progression varied, the type of insertion was classified simply, hence the analysis was not three-dimensional.

In conclusion, the position of a catheter inserted in the upper thoracic epidural space is difficult to predict. Therefore, deep insertion of an epidural catheter should be performed under Carm guidance to check the catheter tip position and presence of coiling.

\section{References}

1. Bromage PR. Continuous epidural anesthesia. In: Epidural Analgesia. Philadelphia, WB Saunders. 1978, pp 215-57.

2. Kim HS, Lim YJ, Kim CS. Epidural catheter length that can be threaded without coiling in lumbar epidural space. Korean J Anesthesiol 1998; 34: 72-6.

3. Bridenbaugh LD, Moore DC, Bagdi P, Bridenbaugh PO. The position of plastic tubing in continuous-block techniques: an x-ray study of 552 patients. Anesthesiology 1968; 29: 1047-9.

4. Ryu HG, Bahk JH, Lee CJ, Lim YJ. The coiling length of thoracic epidural catheters: the influence of epidural approach angle. Br J Anaesth 2007; 98: 401-4

5. Muneyuki M, Shirai K, Inamoto A. Roentgenographic analysis of the positions of catheters in the epidural space. Anesthesiology 1970; 33: 19-24. 